\title{
La preparación de la clase encuentro
}

José Luis Lavín Martínez*

http://dx.doi.org/10.21503/lex.v10i10.229

* Catedrático de la Universidad de Guantánamo (Cuba). Profesor e investigador del Centro de Estudios de Educación Superior (CEDES). Lic. en Psicología por la Universidad Pedagógica José Martí (1985). Lic. en Pedagogía por la Universidad Pedagógica José Martí (1985). Lic. en Cultura Física por la Universidad Pedagógica de Guantánamo (1990). Máster en Ciencias de la Investigación Educativa, Instituto Central de Ciencias Pedagógicas (ICCP), Ministerio de Educación, La Habana, Cuba. jlavinmartinez@fcs. cug.co.cu

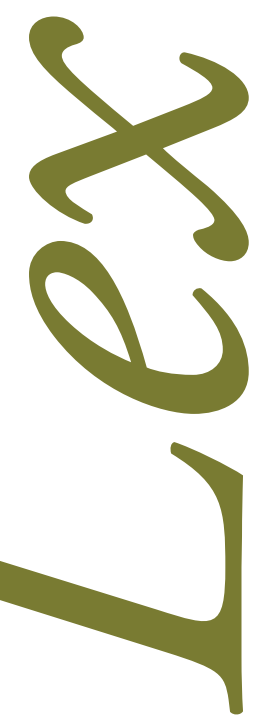





\section{RESUMEN}

En el mundo actual, la clase y los tipos de clases son en algunos casos muy valorados, y en otros, cuestionados por algunos estudiosos del tema; otros no los tienen en cuenta o no los priorizan de acuerdo a sus teorías, paradigmas y enfoques. Hay algunas "escuelas" donde las trabajan priorizando los objetivos, habilidades, capacidades, valores, actitudes, por competencias, etc.

Existen, igualmente, distintos sistemas de organización del proceso docente-educativo: trabajo individual, mesa redondas, talleres, seminarios, conferencias y la clase. Estas y otras formas organizativas se pueden combinar conforme a la estructura y el desarrollo del proceso docente-educativo.

$\mathrm{Al}$ analizar la clase como forma fundamental del proceso docente-educativo, debemos tener presente el punto de vista de la Didáctica, el lugar que ocupa la clase, cómo y en qué situación surge, y cómo se ha ido desarrollando desde el pasado siglo XV hasta nuestros días.

Es necesario valorar que el plan de clase es la herramienta más importante que tiene el profesor para llevar a cabo el proceso docente-educativo con eficiencia y calidad, la guía más eficaz para lograr una buena lección, que en gran medida se logra mediante una buena planificación, diseño y autopreparación de la clase por el docente.

Este tipo de clase encuentro es concebido dentro de las especializadas, y por lo tanto el autor siente el deber de tenerlo presente en este trabajo.

Todo docente debe priorizar la estructura del plan de clase desde el punto de vista metodológico y organizativo en la selección de los contenidos que se van a tratar, la selección de los métodos, procedimientos y otras formas organizativas, además de los medios de enseñanza y audiovisuales más adecuados — los recursos de la tecnología educativa, Web, moddle, entre otros-, las habilidades intelectuales que se trabajarán, los valores morales y sociales, además de las competencias profesionales que deben tributar a la carrera donde se forma el estudiante, de forma tal que todo esté garantizado para cumplir con los objetivos previstos. 
En este trabajo se abordan sugerencias, recomendaciones y consejos útiles para los docentes que no tienen mayor formación pedagógica —entre ellos los noveles—, a fin de que puedan preparar e impartir sus clases si dedican su esfuerzo al estudio y a la autopreparación.

Con esto se da un aporte a la educación de las jóvenes generaciones de educandos que esperan que las actividades docentes se realicen con la calidad que exige el desarrollo de la ciencia, las tecnologías educativas, la didáctica y las diferentes metodologías, sobre todo en la educación superior.

La preparación de la clase consta de tres etapas, en las que se explica, con detalle, cómo el profesor las puede preparar, teniendo en cuenta los infinitivos para formular los objetivos de acuerdo a los niveles de asimilación. El profesor debe observar las tres etapas de la planificación y cumplirlas para alcanzar el éxito esperado.

También se han incorporado algunas recomendaciones y sugerencias para que los profesores tengan las herramientas suficientes a la hora de impartir una buena clase, teniendo en cuenta el tipo, si es combinada o especializada, o la clase encuentro como una clase especializada.

La clase encuentro es una de las formas organizativas del proceso docente educativo que tiene sus propias características ,y como objetivo, la adquisición de conocimientos y habilidades, la formación de intereses cognoscitivos y profesionales en los estudiantes mediante la realización de actividades de carácter esencialmente académico, por encuentro. De ahí el nombre de esta clase.

La estructura de este tipo de clase no está reñida con las de otras clases ya conocidas, pero se deben tener presente algunas cuestiones de orden organizativo y metodológico en el trabajo educativo.

En este tipo de clase es muy importante la selección de los métodos y medios de enseñanzas que están determinados por los objetivos y el contenido, al mismo tiempo que depende de las características de los alumnos, de su edad, su nivel de desarrollo, del dominio o nivel de los conocimientos y habilidades que constituyen antecedentes y la base para el trabajo con la nueva materia, en sus hábitos de trabajo. Esto, desde luego, es muy importante en el profesional que se está formando.

\section{LA PREPARACIÓN DE LA CLASE POR EL DOCENTE}

Todo profesor debe garantizar que en la preparación de sus clases estén presentes las características que les son inherentes a estas, y que la misma responda cada vez en mayor medida a las exigencias de la clase contemporánea, para elevar su nivel de eficiencia en correspondencia con las condiciones y necesidades del desarrollo científico, tecnológico, 
pedagógico y metodológico actual. Es necesario perfeccionar la preparación, así como la planificación adecuada y acertada de la clase.

$\mathrm{Al}$ referirnos a la preparación de la clase, se deben tenerse presente las diferentes etapas en su diseño, cada una de las cuales exigirá más o menos esfuerzo, de conformidad con el nivel de preparación científico-metodológico y docente-metodológico que tenga el docente. Por eso ha de ser una tarea constante de todo pedagogo, el estudio y la participación en todas aquellas actividades que propicien el mejoramiento de su nivel científico y académico.

\section{Etapas que comprende la preparación de la clase}

La preparación de clases comprende tres etapas que se diferencian esencialmente entre sí por sus objetivos.

\section{1) Preparación básica}

Todo profesor debe dominar el o los programas que ha de impartir y conocer cómo estos se proyectan en el currículo o plan de estudios, de manera que pueda identificar los objetivos que pretende cumplir, con el aporte que a los mismos dan los diferentes contenidos, la interrelación que existe entre los contenidos de los programas de cada disciplina, asignatura, curso, semestres y unidades, y por último, la selección adecuada y acertada de los métodos y medios de enseñanza y audiovisuales más adecuados: los recursos de la tecnología educativa, Web, moddle, entre otros.

Junto a los programas que ha de realizar, debe conocer los libros y materiales didácticos que precisan el nivel, extensión y profundidad de los contenidos; las orientaciones metodológicas existentes que facilitan ideas y ejemplos sobre la forma de dirigir la asimilación de los contenidos y, además, los libros de consulta y los cuadernos de actividades que complementan y facilitan el cumplimiento con éxito de una buena clase, que motive, que facilite la comprensión de los contenidos por los alumnos.

Los profesores deben estudiar los documentos normativos y rectores, las orientaciones dadas por las instancias superiores para su vinculación con el contenido de los programas, planes de estudios o currículos de cada carrera, teniendo en cuenta las posibilidades de las disciplinas, asignatura o asignaturas cuyo aprendizaje dirige, así como el nivel de desarrollo de los educandos.

Estos aspectos, que han sido analizados anteriormente, son parte del contenido de trabajo orientado a la preparación de la asignatura anterior al inicio del curso o semestre. 


\section{2) Preparación anterior a la planificación de las clases}

Para que un plan de clases sea eficiente, el docente debe estudiar y diseñar, con la suficiente antelación, el plan de estudios o el currículo de la carrera, el programa de la asignatura, la unidad, el tema, las orientaciones metodológicas, las características de sus alumnos, los contenidos de la básica y complementaria indicada, los textos de consulta y el cuaderno de trabajo que está orientado.

A partir del conocimiento profundo que debe tener de sus alumnos, habrá de precisar cuál será el objetivo como punto de partida para la enseñanza en el trabajo con los contenidos de la unidad y la forma en que debe abordar y desarrollar la misma para obtener el éxito en su trabajo. Para esto tendrá en cuenta los recursos disponibles para el desarrollo del sistema de clases, qué medios están a su alcance y cuáles podrá preparar.

Debe tener presente las actividades prácticas -entre ellas laboratorios, si los lleva- que convengan realizar los alumnos y el profesor, los métodos seleccionados y los medios de enseñanza que se necesitan para cumplir eficientemente con los objetivos propuestos por el programa; es posible que, en algunos casos, esto implique la búsqueda o la elaboración de un medio sustituto, en fin, el hacer uso de la capacidad creadora del docente. Además, el profesor realizará las prácticas previas como parte de su autopreparación para garantizar una eficiente realización de su clase, evitando así la improvisación.

Al desarrollar las actividades prácticas que se realizan en laboratorios, talleres, etc., es importante que el profesor domine los requerimientos que implica la utilización de instrumentos, maquinarias, sustancias, de modo que pueda hacer una utilización óptima de ellos, sin olvidar las normas de protección e higiene necesarias que exige este tipo de actividad.

Para ello, antes de comenzar cada clase, hará un control del estado del taller o laboratorio y lo organizará debidamente, lo cual forma parte de su autopreparación individual. Con esto se evita la improvisación y se hace muy eficiente su actividad docente.

Como parte de su preparación individual, el profesor se incorpora al colectivo de disciplina, año o departamento en el que, bajo la dirección del responsable, se realiza el análisis de la clase diseñada que será impartida y se profundiza en el tratamiento metodológico de cómo serán abordados los contenidos.

Este examen debe partir de la exactitud de las ideas básicas que corresponde disfrutar a los estudiantes para poder abordar el tratamiento de la temática, teniendo en cuenta el nivel real de los educandos, los objetivos del programa, la unidad y su contenido. Se debe analizar, igualmente, la dosificación y tratamiento metodológico, y como consecuencia debe quedar precisado el número de clases, los tipos de clase y los objetivos de cada una de ellas, así como las posibles actividades que durante su desarrollo deben realizar tanto alumnos como profesores. 
Hay que partir del hecho de que cada clase es un eslabón en el sistema de la cadena de clases. Los cambios que van produciéndose en la actividad mental de los educandos, así como los que tienen lugar en la formación de sus convicciones, no se producen instantáneamente sino a través del trabajo continuo del educador. Por ello, las clases tienen que reunir un sistema de criterios científicos, didácticos y metodológicos.

La realización de este trabajo en el marco del colectivo de año o asignatura propicia la discusión de las mejores experiencias docentes y el apoyo a los profesores noveles, sin formación pedagógica y/o con otros perfiles profesionales que se dedican a la docencia o tienen más necesidad de ayuda de los más experimentados, lo que debe contribuir a la elevación de la calidad del trabajo.

En algunas ocasiones, es posible que el tratamiento metodológico de la clase deba completarse con el desarrollo de algún tema sobre el contenido, cuando este tiene un alto grado de complejidad y ofrece dificultades para algunos profesores, o de una clase metodológica instructiva o demostrativa seleccionada entre aquellas que por su contenido o tipo así lo exijan, como parte de la preparación metodológica del docente. Esto puede ser tema para otro artículo que bien vale la pena sea publicado por la revista Educación y Desarrollo.

\section{3) Preparación o diseño de las clases}

Con las orientaciones analizadas anteriormente y teniendo como base esta preparación, cada profesor debe proceder a diseñar los planes de clases.

Esta actividad debe ser individual, por cuanto en su desarrollo el docente debe poner en correspondencia todo lo analizado anteriormente, con las particularidades individuales de sus estudiantes.

En la clase diseñada debe haber una determinación en la selección de los métodos, procedimientos, medios de enseñanzas, la relación de los objetivos con el contenido y las habilidades intelectuales, prácticas profesionales, etc. que se trabajarán durante la clase, de los objetivos que serán evaluados, etc.

Con todo esto se logra motivar mejor a los estudiantes, elevar la capacidad de trabajo, el rendimiento, la adquisición de conocimientos con más calidad, eficiencia en el aprendizaje, y por lo tanto debe tener un carácter creador, científico y técnico.

\section{Observaciones que deben considerarse en la planificación de cada clase}

- Se recomienda que la planificación de las clases se realice de forma sistemática, o sea, no debe diseñarse de forma aislada, pues cada una de ellas constituye un eslabón fundamental en el proceso de enseñanza y el aprendizaje. 
- Cada clase es un eslabón importante en el complejo proceso docente-educativo, cuya unidad tiene un carácter relativo por cuanto, si bien es indispensable que cumpla los objetivos que se propone, esto solo se logra con el aval de los logros alcanzados en clases anteriores (por eso señalamos el carácter de sistema) y porque constituyen la base para la asimilación del nuevo contenido, así como porque los resultados que se obtengan constituyen antecedentes para el logro de objetivos en clases futuras y la relación intermateria y la interdisciplinaridad.

- El deber principal de todo profesor es lograr el aprendizaje de sus estudiantes, para lo cual asume la dirección del proceso a fin de que al final de la clase todos los alumnos hayan alcanzado el mismo nivel y, en lo esencial, los objetivos propuestos.

- La orientación hacia el objetivo tiene que verse como un proceso motivacional, cognoscitivo y regulado que contribuye e influye favorablemente en los resultados alcanzados en el aprendizaje de los estudiantes.

- La dirección adecuada del proceso docente-educativo supone la definición de la situación que ha de crearse en cada momento, de modo que se cumplan las funciones didácticas necesarias, sin perder de vista que es necesario controlar constantemente los resultados alcanzados y que se avance solo cuando el profesor esté seguro de que ha tenido éxito en el logro de lo propuesto.

Determinación de los objetivos. La claridad y precisión que se logre en su determinación condiciona el contenido y su relación con el desarrollo de las habilidades, hábitos, capacidades, destreza y convicciones, la organización y estructura del proceso que se dará a su desarrollo y lo que se deberá controlar para comprobar y valorar la eficiencia del proceso docenteeducativo. Los objetivos están vinculados también con el tipo de clase. Se recomienda que el objetivo esté determinado siempre en función del aprendizaje de los alumnos.

Es preciso, cuando la clase está dirigida esencialmente a propiciar en gran medida el logro de algún objetivo de tipo educativo, que se le incorpore explícitamente en el diseño de la clase. De lo contrario, se entiende que no es indispensable, el trabajo educativo ha de estar presente en todo momento de la clase y de la vida escolar.

\section{EL PLAN DE CLASE}

El plan de clase es el producto de la preparación, diseño, planificación, investigación, estudio y reflexión del profesor sobre la forma más efectiva de desarrollar "su clase". Esta es la guía del docente, y el éxito de ella depende en gran medida de la preparación, experiencia y responsabilidad del pedagogo. Constituye la manera en que el profesor ha decidido organizar y dirigir la actividad del alumno en el desarrollo óptimo de su aprendizaje. 


\section{Funciones importantes del plan de clase}

- Debe recoger en forma abreviada lo que el docente en su preparación ha decidido desarrollar, teniendo como base una idea clara sobre los objetivos que debe cumplir, las habilidades, hábitos y capacidades que debe alcanzar y su relación con el contenido que está trabajando, los métodos que ha de utilizar, los medios de enseñanza, las preguntas que tiene previstas hacer para controlar y evaluar lo que se propone lograr, y la organización que tiene prevista en el proceso que va a seguir para ello.

- Debe servir de guía al profesor para el desarrollo de la misma.

Elplan de clase y las observaciones que el profesor haga sobre su funcionamiento constituyen una importante referencia para la orientación de su trabajo futuro.

Todos los pedagogos necesitan y deben hacer su plan de clase, independientemente de su experiencia (esto constituye una guía que ayuda en buena medida y contribuye al éxito en la enseñanza y el aprendizaje), porque cada clase es una situación nueva, determinada por las condiciones en que se realiza, las características de los alumnos, los medios de enseñanza con que se cuenta y el desarrollo alcanzado por el propio profesor y el educando.

Al concebir el plan de clase encuentro se establecen con precisión el objetivo, el contenido, las habilidades, los hábitos y capacidades que se van a trabajar con los métodos, los medios de enseñanza y la estructura didáctica que se manifiestan en el desarrollo de la misma.

En la parte más importante o principal de la clase encuentro se debe priorizar y concebir lo que no debe faltar, y es lo siguiente:

1. Aclaraciones de dudas sobre lo tratado en el encuentro anterior.

2. Revisión de las actividades de trabajo independiente orientadas por el docente.

3. Control y evaluación de los contenidos orientados en el encuentro anterior.

4. Realizar actividad orientadora de la nueva materia en función de los objetivos generales y específicos; es necesario aclarar que en ese momento se tratarán los conceptos básicos del contenido, para que los estudiantes conozcan las leyes, categorías y premisas fundamentales que se analicen.

5. Orientación del trabajo independiente, con precisiones muy específicas.

6. Relación estricta con los objetivos del tema.

7. Nivel de preferencia de exigencias no reproductivas. 
8. Nivel de aplicación de problemas que estimulen la creatividad, desarrollo intelectual e inteligencia, utilizando métodos problémicos e investigativos.

9. Contribución al desarrollo de habilidades y competencias profesionales.

10. Suficiencia y precisión en las indicaciones bibliográficas que se van a utilizar.

11. Procedimientos para resolver las actividades y orden lógico de realización de las mismas.

12. Análisis de la Guía de Estudio

13. Otras.

Para la orientación del trabajo independiente se deben tener en cuenta las siguientes etapas:

a) Planificación del trabajo independiente.

b) Orientación del trabajo independiente.

c) La orientación para la ejecución del trabajo independiente en la clase y fuera de ella.

d) El control y evaluación del trabajo independiente.

El trabajo independiente precisa de una fuerte motivación, de sistematicidad, de control, de un nivel progresivo de complejidad y de una adecuada orientación por parte del profesor. Se recomienda logicidad por el docente al ser concebida.

En la estructura didáctica de este tipo de clase se precisan contenidos, métodos y procedimientos, medios de enseñanza, el control y la evaluación y las formas para desarrollar y organizar los contenidos.

La selección de los métodos está determinada por el objetivo y el contenido, y al mismo tiempo depende de las características de los alumnos, de su edad, su nivel de desarrollo, del dominio o nivel de los conocimientos, las habilidades que constituyen antecedentes y son base para el trabajo con la nueva materia, de sus hábitos de trabajo, etc. En la selección de métodos y procedimientos, el profesor debe tener presente la importancia de la participación activa y consciente de los alumnos y del trabajo independiente.

Determinación de la estructura de la clase

Se precisan las situaciones que se irán sucediendo, las actividades que deberán desarrollarse en correspondencia con las funciones didácticas analizadas anteriormente.

Valorar que no todas las clases tienen una misma estructura y que es imposible enmarcar su desarrollo en algo " rígido", siempre igual, así como tratar de enmarcar todas las clases 
en esquemas únicos sería negar la naturaleza del proceso docente-educativo. Esto depende bastante de la experiencia del docente y de su nivel de preparación, competencia, iniciativas y sello personal, etc.

Cada tipo de clase supone un énfasis diferente en las distintas funciones didácticas, que frecuentemente se dan unas unidas a las otras, aunque son muy variadas las situaciones docentes que pueden crearse para ajustar la enseñanza a las condiciones en que esta se realiza. No obstante, al desarrollar la clase es oportuno tener en cuenta las observaciones siguientes:

1. En toda clase es necesario asegurar las condiciones básicas para que el alumno pueda apoyarse en lo conocido y "para aprender mejor lo nuevo", yendo de lo fácil a lo difícil, de lo simple a lo complejo.

2. Se debe orientar a los alumnos hacia los objetivos a desarrollar en la clase, informarles sobre qué deben lograr y cómo deberán participar en la misma. Esto favorece su participación consciente y sus posibilidades de asimilación.

3. En toda clase estará presente la comprobación de los resultados, pero el camino para llegar a ello estará en correspondencia con las particularidades de sus alumnos.

4. En el plan de clase se debe considerar la dosificación del tiempo aproximado que se dedicará a sus momentos esenciales. Esto garantiza que se cumplan los objetivos de la forma más eficiente.

5. Se valorará que parte de la clase, de conformidad con los objetivos propuesto, deberá llevar más tiempo, proporcionalmente a las necesidades, es decir, que la duración se distribuirá entre los distintos momentos fundamentales de la clase.

Es preciso tener presente que el plan de la clase encuentro no es el resumen de la clase, no es una suma de notas tomadas del libro de texto o las orientaciones metodológicas; es el conjunto de líneas de acción, de pasos metodológicos y lógicos en que se apoyará el profesor para evitar improvisaciones en la clase. Hay algunos profesores con mucha experiencia que lo llaman el "guión" que el docente debe tener.

Esta guía debe ser consultada constantemente en el desarrollo de la clase, pues de esta manera se evitan pérdidas de tiempo. Por ello contiene las preguntas fundamentales que debe hacer al dirigir la actividad, los ejercicios o actividades que no aparecen en el libro de texto, ni en el cuaderno de trabajo que deberán realizar todos o algunos estudiantes. En este caso, bastaría precisar la fuente, página y número de los ejercicios. Esto último se recomienda que aparezca en el plan de clases. 


\section{Relación entre el plan de la clase y su desarrollo}

El plan de clase constituye un documento de carácter obligatorio y fundamental para su desarrollo, pero no es en sí la clase.

El éxito de la clase está dado, en definitiva, por la puesta en práctica del plan de clase en forma viva y creadora, en correspondencia con la realidad de cada instante, con la respuesta que se logre en los alumnos ante sus exigencias y, como se ha venido enfatizando, en el trabajo de las habilidades y competencias profesionales que posee el docente.

Además, hay un factor que no está recogido en el plan y cuya presencia en la clase es fundamental: el factor socioemocional.

Este factor está presente en la interrelación profesor-alumno y en el logro de la formación de intereses, sentimientos, actitudes. Cuando el pedagogo no es capaz de establecer las relaciones adecuadas con sus alumnos, de responder a sus necesidades, motivaciones y valoraciones, esto se traduce en la falta de participación y desinterés, en fracaso en el rendimiento y rechazo a la asignatura, el tema o al propio docente.

Una buena clase constituye los cimientos para esa gran obra que es el proceso pedagógico, cuyo objetivo fundamental es formar a las generaciones del mañana, los hombres y mujeres de cualquier país que aspire al desarrollo. 


\section{REFERENCIAS}

- Álvarez de Zayas, Carlos. Hacia una escuela de excelencia. Cuba, La Habana: Editorial Academia, 1998.

La didáctica como ciencia. Su objeto. Los objetivos de la enseñanza. Enfoque sistémico de la didáctica en la Educación Superior cubana. La Habana, Cuba, 1999.

La escuela en la vida. La Habana, Cuba, 2000.

"Características esenciales pedagógicas de la escuela cubana". Revista Educación. La Habana, No. 100, mayo-agosto, 2000, Segunda época.

- Añorga Morales, Julia. Pedagogía y estrategia-didáctica curricular de educación avanzada. La Habana, Cuba: ISP, Enrique J. Varona, 1977.

- Bermúdez Sarguera, Rogelio y Rodríguez Rebustillo, Marisela. Teoría y metodología del aprendizaje. Ciudad de la Habana: Editorial Pueblo y Educación, 1996.

- Danilov, M. A. y Skatkin, M.N. Didáctica de la escuela media. Ciudad de La Habana: Editorial Pueblo y Educación, 1978.

- Didáctica de la Escuela Media. La Habana, Cuba: Editorial libros para la Educación, 1980.

- Diccionario de la lengua española y de nombres propios. España: Editorial Océano, S.A.

- Diccionario Enciclopédico Grijalbo. España: Océano Grupo Editorial, S.A., 1999.

- Domínguez Pino, Martha. La Habana, Cuba: Editorial Pueblo y Educación, 2004.

- Colectivo de autores, La didáctica y los métodos científicos generales de investigación. La Habana: Editorial Ciencias Sociales, 1985.

- Colectivo de autores. Pedagogía. Ciudad de La Habana: Editorial Pueblo y Educación, 1984.

- Colectivo de autores (material mimeografiado). Las habilidades de carácter intelectual. 1977.

- Colectivo de autores. Compendio de Pedagogía. Ciudad de La Habana, Cuba: Editorial Pueblo y Educación, 2002.

- López López, Mercedes. Saber enseñar a describir, definir, argumentar. La Habana, Cuba: Editorial Pueblo y Educación, 1990. 
- López, M. y Pérez, Celia. La dirección de la actividad cognoscitiva. Colección Biblioteca del Director, Ciudad de La Habana: Editorial Pueblo y Educación, 1997.

- Morales Martínez José M. Teoría y metodología de la educación fisica. La Habana, Cuba: Ed. Producción de Medios de Enseñanza, 1976.

- Ministerio de Educación. Primer Seminario Nacional a dirigentes, metodólogos e inspectores de las direcciones provinciales y municipales de educación. Primera, segunda y tercera parte. La Habana, enero de 1977.

Segundo Seminario Nacional a dirigentes, metodólogos e inspectores de las direcciones provinciales y municipales de educación. Tema II, Ciudad de La Habana: Empresa de Impresiones Gráficas, 1977.

Tercer Seminario Nacional a dirigentes, metodólogos e inspectores de las direcciones provinciales y municipales de educación. Tema III, Ciudad de La Habana: Empresa de Impresiones Gráficas, 1979.

Cuarto Seminario Nacional a dirigentes, metodólogos e inspectores de las direcciones provinciales y municipales de educación. Temas IV y V, Ciudad de La Habana, Cuba: Emp. de Impresiones Gráficas, 1981.

- Pita, B. y C. Pérez. La clase, seminario a dirigentes y metodólogos. Documentos Normativos, La Habana, Cuba: Editorial Antonio Valido, MINED, 1977.

- Tomaschewski. K. Didáctica general. La Habana, Cuba: Editorial de Libros para la Educación, 1978.

- Fuentes González, Homero. Fundamentos didácticos para un proceso de enseñanzaaprendizaje participativo. Santiago de Cuba, C.E.E.S “Manuel F. Gran”: Universidad de Oriente, 1997.

$-$ C.E.E.S. "Manuel F. Gran"; Universidad de Oriente, 1997.

Conferencia de Didáctica del Postgrado. Santiago de Cuba, Didáctica de la Educación Superior.

"Aprendizaje e instrucción", en: Compendio de lectura sobre currículum: diseño, práctica y evaluación. Curso internacional. La Habana: CEPES, 1994, $2 \mathrm{t}$.

- Gumuman, V. E.; Korolev. V. F. Pedagogía. La Habana: Cuba: Ed. Pueblo y Educación, 1967.

- González Castro, Vicente. Medios de enseñanza. La Habana, Cuba: Ed. Pueblo y Educación, 1979. 
- Silvestre Oramas, Margarita y Zilberstein Torucha, José. Hacia una didáctica desarrolladora. Colectivo de autores del MINED.

- Pedagogía. Ciudad de La Habana, Cuba: Editorial Pueblo y Educación, 2002.

- Shubin. Elplan declase del maestro. La Educación por el mundo, Centro de Documentación e Información Pedagógica, ICCP, 1981.

- Tonkonogaya, E. P., Didakticheskiye Trioeboraniya K. Shkolie. Exigencias didácticas para la clase en la escuela nocturna. Leningrado, 1969.

- Talizina, N. Psicología de la enseñanza. Moscú: Editorial Progreso, 1988.

- Vejler, S. "El análisis psicológico y pedagógico de la clase", en: Revista de Instrucción Pública No. 6, 1975.

- Yacoliev, Nicolai. Metodología y técnica de la clase. Ciudad de La Habana: Editorial Libros para la Educación, 1979.

- Ortiz Torres Emilio y María de los Ángeles Mariño Sánchez. Revista Pedagogía Universitaria. Vol. 9, No. 1, 2004.

Internet, 2005.

- Petrovich Baranov, Serguei. Didáctica de la Escuela Primaria. Ciudad de La Habana: Editorial Pueblo y Educación, 1990.

- Klingberg, Lothart, Introducción a la Didáctica. Ciudad de La Habana: Editorial Pueblo y Educación, 1978. 


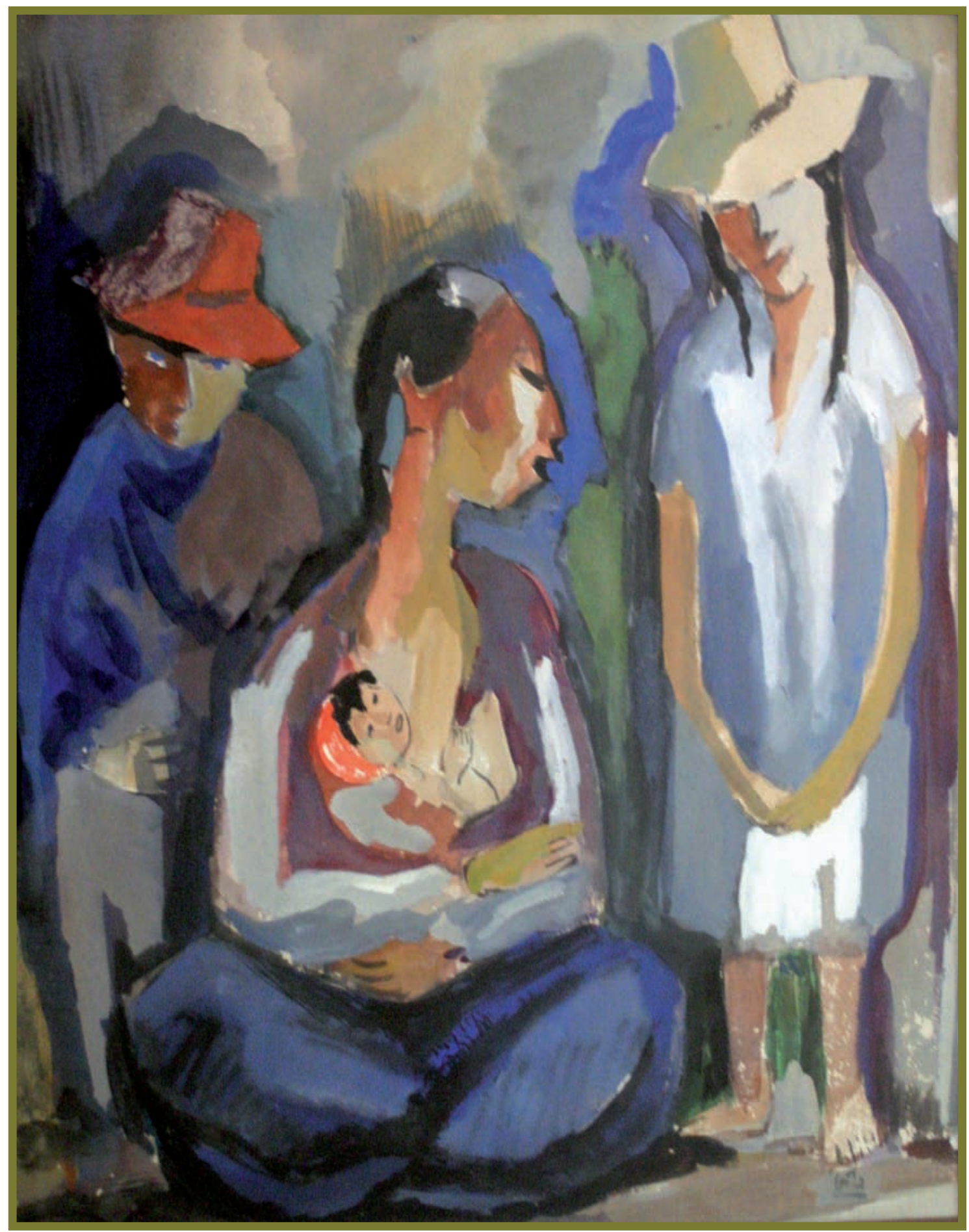

Maternidad II. 